\title{
ON BOUNDEDNESS OF SOLUTIONS OF SECOND ORDER DIFFERENTIAL EQUATIONS IN THE LIMIT CIRCLE CASE
}

\author{
MAN KAM KWONG
}

ABSTRACT. A differential equation of the form $x^{\prime \prime}(t)+a(t) x(t)=0$, $t \geq 0$, is said to be in the limit circle case if all its solutions are square integrable on $[0, \infty)$. It has been conjectured in [1] that all its solutions are bounded. J. Walter recently gave a counterexample. This paper gives a method of modifying any given equation in the limit circle case with bounded solutions to produce one with unbounded solutions.

1. Introduction. H. Weyl [2] has classified the one-dimensional wave equation

$$
x^{\prime \prime}(t)+a(t) x(t)=0, \quad t \geq 0,
$$

where $a(t)$ is a locally integrable function defined on $[0, \infty)$, as

Limit circle case - when all solutions of (1) belong to $L^{2}[0, \infty)$.

Limit circle case - when at least one solution is not square integrable on $[0, \infty)$.

Such a classification is closely related to the study of selfadjoint boundary value problems and more generally in the study of the spectra of second order differential operators.

H. Weyl has obtained the result that if (1) is in the limit circle (or limit point) case, then the following perturbed equation

$$
x^{\prime \prime}(t)+(a(t)+b(t)) x(t)=0,
$$

where $b(t)=O(1)$ is a bounded real-valued function, is still in the limit circle (or limit point) case.

J.S.W. Wong and W. T. Patula in [1] raise the conjecture that Weyl's result is still true when the condition $b(t)=O(1)$ is replaced by $b(t) \epsilon$ $L^{p}[0, \infty)$, for some $p \geq 1$. By a decomposition device [3], Wong is able to reduce the problem to the case when $p=1$. Yet the conjecture remains open, and only partial results have been established. Many of the known examples of limit circle case furnish bounded solutions. Besides, in [1] the following theorem was proved.

Theorem (Patula and Wong). Suppose that all solutions of (1) are bounded. If equation (1) is in the limit circle case, then equation (2) is also in the limit circle case and all of its solutions are also bounded.

Received by the editors June 26, 1974 .

AMS (MOS) subject classifications (1970). Primary 34B05.

Key words and phrases. Limit circle case, unbounded solutions. 
This theorem suggested the following

Conjecture. If equation (1) is in the limit circle case, then all its solutions must necessarily be bounded.

The hope was that once this conjecture is answered in the affirmative, the theorem cited above would settle completely the former conjecture generalizing Weyl's result.

However, Johann Walter gave the following counterexample: The function

$$
x(t)=g(t) \cos \left(\int_{0}^{t} g^{-2}(s) d s\right),
$$

where $g(t)$ is any $C^{2}$ positive function, satisfies an equation of the form (1) with $a=g^{-4}-g^{\prime \prime} g^{-1}$. If $g$ is square integrable, (1) will be in the limit circle case. If furthermore $g$ is a suitably chosen unbounded function, then $x$ is unbounded.

In this paper we shall develop another method by which one can modify any given equation in the limit circle case with bounded solutions to one with unbounded solutions.

2. Main results. Let $x_{1}(t)$ and $x_{2}(t)$ be the solutions of (1) such that $x_{1}(0)=1, x_{1}^{\prime}(0)=0, x_{2}(0)=0, x_{2}^{\prime}(0)=1$. We call $\left\{x_{1}(t), x_{2}(t)\right\}$ the fundamental set of solutions of (1). Notice that their Wronskian, $\left[x_{1}(t) x_{2}^{\prime}(t)-\right.$ $\left.x_{1}^{\prime}(t) x_{2}(t)\right]$, is constantly equal to 1 . Also notice that $(1)$ is in the limit circle case if and only if $\int_{0}^{\infty}\left[x_{1}^{2}(t)+x_{2}^{2}(t)\right] d t<\infty$.

Lemma 1. Let $F(t)$ be a $C^{2}$ function defined on an interval $[\alpha, \beta]$ so that $F(t) \geq m$, for some positive real number $m$, and for all $t$ in the interval. $t_{0}$ is a given point in $[\alpha, \beta]$. Then we can construct another function $G(t)$ with the following properties:

(A) $G(t) \geq m$ for all $t$ in $[\alpha, \beta] ; G\left(t_{0}\right)=m$;

(B) $G(t)$ is a $C^{2}$ function and $G(t)=F(t), G^{\prime}(t)=F^{\prime}(t), G^{\prime \prime}(t)=F^{\prime \prime}(t)$, for $t=\alpha$ or $\beta$;

(C) $\int_{a}^{\beta} G(t) d t=\int_{a}^{\beta} F(t) d t$.

Proof. The proof is elementary.

Lemma 2. Let $\left\{x_{1}(t), x_{2}(t)\right\}$ be the fundamental set of solutions of (1). $[\alpha, \beta]$ is an interval in which $x_{1}(t)>0$. Construct another $C^{2}$ function $y_{1}(t)$ with the following properties:

(i) $y_{1}(t)=x_{1}(t)$ for $t \in[0, \infty)-(\alpha, \beta)$;

(ii) $y_{1}(t)>0$ for $t \in[\alpha, \beta]$;

(iii) $\int_{\alpha}^{\beta} d t / x_{1}^{2}(t)=\int_{a}^{\beta} d t / y_{1}^{2}(t)$.

Then $y_{1}(t)$ is one of the fundamental solutions of the following equation:

$$
y^{\prime \prime}(t)+a_{1}(t) y(t)=0, \quad t \geq 0,
$$

in which 


$$
a_{1}(t)=a(t) \text { for } t \in[0, \infty)-(\alpha, \beta) .
$$

If $y_{2}(t)$ is another solution of $\left(1^{*}\right)$ satisfying the initial conditions $y_{2}(0)$ $=0$, and $y_{2}^{\prime}(0)=1$, then

$$
y_{2}(t)=x_{2}(t) \quad \text { for } t \in[0, \infty)-(\alpha, \beta) .
$$

Proof. (3) is a consequence of (i).

In the interval $[0, \alpha]$, equations $(1)$ and $\left(1^{*}\right)$ coincide. Furthermore, $x_{2}(t)$ and $y_{2}(t)$ have the same initial conditions at $t=0$, implying that they coincide in $[0, \alpha]$. From the equality $x_{1} x_{2}^{\prime}-x_{1}^{\prime} x_{2}=1$, and the fact that $x_{1}(t)>0$ in $[\alpha, \beta]$, we obtain

$$
\frac{d}{d t}\left(\frac{x_{2}(t)}{x_{1}(t)}\right)=\frac{1}{x_{1}^{2}(t)}
$$

and so

$$
\frac{x_{2}(t)}{x_{1}(t)}-\frac{x_{2}(\alpha)}{x_{1}(\alpha)}=\int_{\alpha}^{t} \frac{d s}{x_{1}^{2}(s)}
$$

or

$$
x_{2}(t)=x_{1}(t)\left[\frac{x_{2}(a)}{x_{1}(a)}+\int_{a}^{t} \frac{d s}{x_{1}^{2}(s)}\right]
$$

for all $t \in[\alpha, \beta]$. The same reasoning when applied to $y_{1}(t)$ and $y_{2}(t)$ gives

$$
y_{2}(t)=y_{1}(t)\left[\frac{y_{2}(\alpha)}{y_{1}(\alpha)}+\int_{a}^{t} \frac{d s}{y_{1}^{2}(s)}\right] .
$$

Now it follows from (4) and (5), when (iii) and the equalities $y_{1}(\alpha)=x_{1}(\alpha)$, $y_{2}(\alpha)=x_{2}(\alpha)$ and $y_{1}(\beta)=x_{1}(\beta)$ are taken into account, that $y_{2}(\beta)=x_{2}(\beta)$.

Differentiation of (4) gives

$$
x_{2}^{\prime}(\beta)=x_{1}^{\prime}(\beta)\left[\frac{x_{2}(\alpha)}{x_{1}(\alpha)}+\int_{\alpha}^{\beta} \frac{d s}{x_{1}^{2}(s)}\right]+\frac{1}{x_{1}(\beta)} .
$$

A similar formula for $y_{2}^{\prime}(\beta)$ shows that $x_{2}^{\prime}(\beta)=y_{2}^{\prime}(\beta)$. The equations (1) and $\left(1^{*}\right)$ coincide in $[\beta, \infty)$, while $x_{2}(t)$ and $y_{2}(t)$ are their solutions with the same initial conditions at $\beta$. Hence they must also be equal in $[\beta, \infty)$. This completes the proof.

The geometrical significance of Lemma 2 is that given equation (1), we can modify the function $a(t)$ within a given interval $[\alpha, \beta]$ to obtain equation $\left(1^{*}\right)$, so that the two fundamental sets of solutions of $(1)$ and $\left(1^{*}\right)$ only differ within $[\alpha, \beta]$. We are going to apply this technique to alter $(1)$ to $\left(1^{*}\right)$ so that 
$\left(1^{*}\right)$ will have a solution that attains a sufficiently large value.

Lemma 3. Let $x_{1}(t)$ be the solution of (1) as defined above. Suppose that $x_{1}\left(t_{0}\right)>0$ for some $t_{0}$. It is possible to alter equation (1) in an interval $[\alpha, \beta]$ which is as small as we please and which contains $t_{0}$ so that the fundamental sets of solutions of (1) and $\left(1^{*}\right)$ differ only in $[\alpha, \beta]$ and

(A) $y_{1}\left(t_{0}\right) \geq M$,

(B) $\int_{a}^{\beta} y_{1}^{2}(t) d t<\epsilon$ and $\int_{a}^{\beta} y_{2}^{2}(t) d t<\epsilon$

where $M$ and $\epsilon$ are preassigned positive real numbers.

Proof. If $x_{1}\left(t_{0}\right) \geq M$, we simply do not have to alter (1). So we assume that $x_{1}\left(t_{0}\right)<M$. It is possible to choose an interval $[\alpha, \beta]$ containing $t_{0}$, so small that

(i) $0<x_{1}(t)<M$ for all $t \in[\alpha, \beta]$,

(ii) $\int_{a}^{\beta} d t / x_{1}^{2}(t)<1$,

(iii) $2 M^{2}\left[\left(x_{2}(\alpha) / x_{1}(\alpha)\right)^{2}+1\right](\beta-\alpha)<\epsilon$.

Now apply Lemma 1 to the function $F(t)=x_{1}^{-2}(t)$ with $m=M^{-2}$ to obtain $G(t)$. Define

$$
y_{1}(t)= \begin{cases}x_{1}(t), & t \in[0, \infty)-(\alpha, \beta), \\ G(t)^{-1 / 2}, & t \in(\alpha, \beta) .\end{cases}
$$

Then $y_{1}(t) \leq M$ for $t \in[\alpha, \beta]$ and $y_{1}\left(t_{0}\right)=M$. The first inequality in (B) follows from (iii). The fact that $F(t)=G(t), F^{\prime}(t)=G^{\prime}(t)$, and $F^{\prime \prime}(t)=G^{\prime \prime}(t)$ at $t=\alpha$ and $\beta$ implies that $y_{2}(t)$ is $C^{2}$. Lemma 2 shows that $y_{2}(t)$ and $x_{2}(t)$ differ only in $[\alpha, \beta]$. Expression (5) shows that

$$
\begin{gathered}
y_{2}^{2}(t) \leq 2 y_{1}^{2}(t)\left[\left(\frac{y_{2}(\alpha)}{y_{1}(\alpha)}\right)^{2}+\left(\int_{a}^{t} \frac{d s}{y_{1}^{2}(s)}\right)^{2}\right] \leq 2 y_{1}^{2}(t)\left[\left(\frac{x_{2}(\alpha)}{x_{1}(\alpha)}\right)^{2}+\left(\int_{\alpha}^{\beta} \frac{d s}{y_{1}^{2}(s)}\right)^{2}\right] \\
\leq 2 M^{2}\left[\left(\frac{x_{2}(\alpha)}{x_{1}(\alpha)}\right)^{2}+1\right] \Rightarrow \int_{\alpha}^{\beta} y_{2}^{2}(t) d t \leq \epsilon \quad \text { (by (iii)). }
\end{gathered}
$$

Theorem. Given any equation of the form (1) in the limit circle case, it is possible to alter $a(t)$ to $a_{1}(t)$ so that $\left(1^{*}\right)$ is still in the limit circle case but one solution of $\left(1^{*}\right)$ is unbounded.

Proof. Choose a sequence of points $\left\{t_{n}\right\}$ which increases to infinity, so that $x_{1}\left(t_{n}\right)>0$ for all $n$. By the construction given in Lemma 3 , it is possible to alter $a(t)$ in a sequence of intervals $\left\{\left[\alpha_{n}, \beta_{n}\right]\right\}$ which are pairwise disjoint and each contains $t_{n}$ so that $y_{1}\left(t_{n}\right) \geq n$, while

$$
\int_{a_{n}}^{\beta_{n}} y_{1}^{2}(t) d t<\frac{1}{2^{n}} \text { and } \int_{a_{n}}^{\beta_{n}} y_{2}^{2}(t) d t<\frac{1}{2^{n}}
$$


Furthermore, $y_{1}(t)$ and $y_{2}(t)$ differ from $x_{1}(t)$ and $x_{2}(t)$ only in $\bigcup_{n=1}^{\infty}\left[\alpha_{n}, \beta_{n}\right] .\left(1^{*}\right)$ is still in the limit circle case because

$$
\begin{aligned}
\int_{0}^{\infty} y_{i}^{2}(t) d t & =\int_{A} y_{i}^{2}(t) d t+\sum_{n=1}^{\infty} \int_{a_{n}}^{\beta_{n}} y_{i}^{2}(t) d t \\
& \quad\left(\text { where } A=[0, \infty)-\bigcup_{n=1}^{\infty}\left[a_{n}, \beta_{n}\right], i=1,2\right) \\
< & \int_{A} x_{i}^{2}(t) d t+\sum_{n=1}^{\infty} \frac{1}{2^{n}} \leq \int_{0}^{\infty} x_{i}^{2}(t) d t+1<\infty .
\end{aligned}
$$

\section{REFERENCES}

1. W. T. Patula and J. S. W. Wong, An $L^{p}$-analogue of the Weyl alternative, Math. Ann. 197 (1972), 9-27. MR 45 \#8913.

2. H. Weyl, Über gewohnliche Differentialgleichungen mit Singularitäten und die zugehorige Entwicklung willkurlicher Funcktionen, Math. Ann. 68 (1910), 220269.

3. J. S. W. Wong, Square integrable solutions of $L^{p}$ perturbations of second order linear differential equations (to appear).

DEPARTMENT OF MATHEMATICS, HONG KONG BAPTIST COLLEGE, KOWLOON, HONG KONG 